\title{
Luteolin-rich chrysanthemum flower extract suppresses baseline serum uric acid in Japanese subjects with mild hyperuricemia
}

\author{
Marina Hirano ${ }^{1}$, Shogo Takeda ${ }^{1}$, Shoketsu Hitoe ${ }^{1}$, and Hiroshi Shimoda ${ }^{* 1}$ \\ ${ }^{1}$ Oryza Oil \& Fat Chemical Co. Ltd., 1 Numata, Kitagata-cho, Ichinomiya, Aichi, 493-8001, Japan
}

\begin{abstract}
Background: Luteolin is a flavonoid found in various edible plants that exhibits diverse health benefits, including anti-inflammatory and anti-gout effects. However, there has been little clinical investigation of luteolin from the viewpoint of gout prevention. We conducted a clinical trial of supplementation with chrysanthemum flower extract rich in luteolin (LCE) to assess the effect on serum uric acid levels in Japanese men.

Methods: We examined the effect of LCE containing 10\% luteolin in two double-blind placebo-controlled studies. In the single-dose study, fasting subjects took a capsule containing $100 \mathrm{mg}$ of LCE (10 mg of luteolin) or placebo before ingestion of a high purine base test meal and the serum uric acid level was determined over time. For the repeated-administration study, the subjects ingested placebo or LCE capsule for 4 weeks. Fasting serum uric acid was evaluated before and after ingestion.
\end{abstract}

Results: Oral intake of LCE had no significant influence on serum uric acid. After 4 weeks of LCE ingestion, serum uric acid tended to decrease in the LCE group. A significant decrease of serum uric acid was observed after LCE ingestion in the subjects with a baseline uric acid level of 5.5 to $7.0 \mathrm{mg} / \mathrm{dL}$. There were no abnormalities suggesting adverse effects during or after ingestion of LCE.

Conclusion: Ingestion of LCE for 4 weeks reduced the serum uric acid level. Luteolin may be able to prevent gout by controlling uric acid.

\begin{abstract}
Abbreviations: XOD: xanthine oxidase; LCE: luteolin-rich chrysanthemum flower extract; CRP: C-reactive protein; AUC: area under the concentration vs. time curve; CFO: chrysanthemum flower oil

Backgrounds

Gout is a disorder in which accumulation of needle-shaped uric acid crystals in peripheral tissues due to hyperuricemia [1]. Nonsteroidal anti-inflammatory drugs [2], steroids [3], and colchicine [4] are prescribed to suppress acute pain and inflammation, while xanthine oxidase (XOD) inhibitors such as allopurinol are used for reduction of high blood uric acid levels [5]. Several Japanese foods have a high content of purine bases, such as nori (seaweed), shiitake (mushrooms), chicken liver, and sakura shrimp [6]. However, Japanese people tend to be more conscious about the purine base content of beer and alcoholic beverages, in spite of it being lower than in the foods mentioned above [7]. Approximately $30 \%$ of Japanese men have hyperuricemia and $0.8 \%$ of outpatients are on treatment for gout [8].
\end{abstract}

Dried chrysanthemum flowers are used as a traditional remedy for gout in China [9]. Several constituents of the flowers exhibit antiinflammatory activity, including triterpene alcohol [10,11], octulosonic acid derivatives [12], and flavonoids [13]. Moreover, an oil containing $1 \%$ chrysanthemum flower extract polyphenol has been reported to suppress dietary hyperuricemia in rats [14]. Luteolin is a flavonoid with 4 hydroxyl groups and is found in a wide variety of edible plants, including chrysanthemum flowers [15]. It has been reported to exhibit diverse biological activities. In relation to gout, luteolin competitively inhibits XOD activity with a micromolar IC50 value [16-18]. Its inhibitory activity against XOD has been confirmed in mice after oral ingestion. In addition, luteolin suppressed enhancement of hepatic XOD activity enhanced by oxonate which induces hyperuricemia [19, 20]. Moreover, luteolin suppresses nitric oxide production by uric acid in pancreatic $\beta$-cells, which exacerbates diabetic complications [21]. However, there is only one clinical report about the influence of luteolin on the blood uric acid level [22]. Therefore, we investigated the effects of luteolin-rich chrysanthemum flower extract (LCE) in Japanese men with slightly high uric acid levels. In the first study, we investigated whether a single oral dose of LCE suppressed the postprandial increase of uric acid in subjects ingesting a diet with a high purine base content. We also investigated whether repeated ingestion of LCE could suppress the serum uric acid level.

\section{Materials and methods}

\section{Subjects}

For the single-dose study, 39 male employees of Oryza Oil \& Fat Chemical Co. Ltd. aged 22 to 71 years were recruited. After receiving

Correspondence to: Hiroshi Shimoda, Oryza Oil \& Fat Chemical Co. Ltd., 1 Numata, Kitagata-cho, Ichinomiya, Aichi, 493-8001, Japan, E-mail: kaihatsu@ mri.biglobe.ne.jp

Key words: luteolin, chrysanthemum, gout, uric acid, purine base

Received: March 10, 2017; Accepted: March 27, 2017; Published: March 30, 2017 
a full explanation of the study purpose and protocol, the candidate subjects agreed to a screening test, which involved intake of a high purine base test meal. Disodium 5'-guanylate (1.62 g, Cheil Jedang Indonesia PT) and sodium 5'-isosiate (1.63 g, Ajinomoto, Japan) were mixed with commercially available powdered corn soup (17.6 g, Knoll cup soup, Ajinomoto, Japan). Then $180 \mathrm{~mL}$ of hot water was added to dissolve the mixture and it was used as the high purine base test meal. The candidate subjects fasted from 10:00 pm on the night before the test and a blood sample was collected on the morning of the test. Then they ingested the test meal and blood samples were collected after 60 $\mathrm{min}, 120 \mathrm{~min}$, and $240 \mathrm{~min}$. Subjects were enrolled if the fasting serum uric acid level was 5.5 to $8.0 \mathrm{mg} / \mathrm{dL}$ and an increase of serum uric acid after ingestion of the test meal was confirmed. There were 20 subjects selected and they were aged from 22 to 70 years old.

For the repeated-administration study of LCE, healthy men aged 20 years or older were recruited from among the employees of Oryza Oil \& Fat Chemical Co. Ltd. who freely gave consent to the study. Among them, 39 men aged 22 to $71(42.8 \pm 13.3)$ years who did not meet any of the "exclusion criteria" below were selected as candidates. After confirmation of informed consent, fasting blood was collected to measure the baseline serum uric acid value, and then 30 participants were selected in order from the highest to lowest uric acid level. The subjects were grouped so that the average age was similar in each group. Uric acid levels ranged from 4.5 to $7.8 \mathrm{mg} / \mathrm{dL}(6.0 \pm 0.9 \mathrm{mg} / \mathrm{dL})$. Exclusion criteria were as follows.

1) Current medication for any chronic symptom.

2) Severe allergy to foods or medicines.

3) Current treatment for hyperuricemia or gout.

\section{Preparation and allocation of test samples}

LCE was obtained by aqueous ethanol extraction from the flowers of Chrysanthemum indicum cultivated in China and powdered by Oryza Oil \& Fat Chemical Co. Ltd. LCE (commercial name: Kiku Flower Extract-P; Lot. T-519) contained 10\% luteolin, and $100 \mathrm{mg}$ of LCE was placed into colored hard capsules for ingestion. Placebo capsules with the same appearance as the LCE capsules that contained $100 \mathrm{mg}$ of dextrin were also made. The capsules were colored and the filling was not visible from outside. The allocation sheet indicating test sample allocation was strictly protected by a controller who was not directly involved in the study.

For the single-dose study, the 20 subjects were allocated to 2 groups so that the average age and uric acid level were similar. For the repeatedadministration study, the 30 subjects were allocated to 2 groups from the highest value to lowest uric acid value.

\section{Protocol}

The studies and statistical analysis were carried out at Oryza Oil $\&$ Fat Chemical Co. Ltd. The studies were done as placebo-controlled double-blind cross-over trials. The primary outcome was the serum uric acid level in both studies.

For the single-dose study (performed on July 8, 2016), one group $(n=10)$ ingested a placebo capsule and the other group ingested an LCE capsule $(\mathrm{n}=10)$. All subjects fasted from 10:00 pm on the night before the test and the first blood collection was carried out in the morning of the test day. Then the subjects ingested the high purine base test meal and the second, third, and fourth blood collections were performed after 60, 120, 180 and $240 \mathrm{~min}$. Urine was collected $2 \mathrm{hr}$ after intake of the test meal. At seven days after the first examination, the same test was repeated. The subjects in each group ingested the other type of capsule and the test meal, and blood was collected in a similar way to that described above.

For the repeated-administration study, blood and urine were collected from fasting subjects in both groups on September 6, 2016. The subjects in each group $(\mathrm{N}=15)$ ingested one placebo capsule or one LCE capsule daily with water after a meal for 4 weeks. Then blood and urine samples were collected from fasting subjects. After a 13-day washout period, blood and urine samples were collected from the fasting subjects again. Then the subjects in each group ingested the other type of capsule (LCE or placebo) for 4 weeks, after which blood and urine samples were collected under fasting conditions.

\section{Blood and urine analysis}

In the single-dose study, the collected blood samples were centrifuged to obtain serum. Uric acid levels in serum and urine were determined by FALCO Biosystems Ltd. (Kyoto, Japan).

In the repeated-administration study, serum was obtained for determination of uric acid and C-reactive protein (CRP), while whole blood was used for measurement of the erythrocyte sedimentation rate and white blood cell count. These parameters were determined by FALCO Biosystems Ltd. Urine $\mathrm{pH}$ was measured by $\mathrm{pH}$ papers ranged from 3.8 to 5.4 and 5.5 to 9.0 .

\section{Ethics and compliance}

This study was performed in accord with the WMA Declaration of Helsinki (64th WMA General Assembly in 2013, Brazil) and was carried out in line with ethical considerations. Each subject's human rights and safety were taken into consideration. The ethics committee of Oryza Oil \& Fat Chemical was made up of scientists from Oryza Oil \& Fat Chemical Co. Ltd. with doctoral degrees in pharmacy, as well as a medical doctor and a lawyer from a legal firm. The committee was convened to assess the ethical nature and appropriateness of the protocol. The two studies were implemented based on the protocol approved by the ethics committee and any substantial deviations from the protocol required authorization by the committee. Both studies were registered with the University Hospital Medical Information NetworkClinical Trial Registry (UMIN000023818 and UMIN000022823).

\section{Investigation of adverse events}

Evaluation of adverse events was carried out and the causal relationship with the test substances was determined. The decision as to whether or not the study should continue was made by the doctor in charge, if necessary. Adverse events were defined as symptoms resulting from ingestion of LCE that were unpleasant for the subject. If a subject asked to discontinue the study, it was promptly stopped and consideration was taken to prevent any disadvantage to the subject.

\section{Exclusion criteria}

If any of the following events occurred during the repeatedadministration study, the case was discussed at the clinical conference and the subject in question was excluded from analysis after review.

1) If the subject was late when participating in each test.

2) If the number of days with no intake of the test product (i.e., the specified daily amount for ingestion was not reached) was greater than $15 \%$ of the total number of planned ingestion days. 
3) If there were major issues with regard to reliability of the data due to problems with testing or other reasons.

4) Other reasons suggesting that it was appropriate to exclude the subject.

\section{Statistical analysis}

The results are reported as the mean and SD (except urine parameters). A two-tailed paired $t$-test was used for comparisons between before and after ingestion of capsules. The unpaired $t$-test was used to compare the placebo group with the LCE groups. A probability of less than $5 \%$ was considered significant.

\section{Results}

\section{Single-dose study}

The results of the tests performed before and after the washout period were combined ( 20 subjects in total). The serum uric acid level before ingestion of the placebo or LCE capsules was $6.8 \pm 1.0 \mathrm{mg} / \mathrm{dL}$ and $6.6 \pm 1.0 \mathrm{mg} / \mathrm{dL}$, respectively. At $2 \mathrm{hr}$ after the test meal ingestion, serum uric acid rose to $9.1 \pm 1.3 \mathrm{mg} / \mathrm{dL}$ (placebo) and $9.0 \pm 1.3 \mathrm{mg} / \mathrm{dL}$ (LCE). Uric acid remained at more than $8.5 \mathrm{mg} / \mathrm{dL}$ at $4 \mathrm{hr}$ after the test meal. The area under the concentration vs. time curve (AUC) was almost the same in both groups, being $457 \pm 179 \mathrm{mg} / \mathrm{dL} \cdot 4 \mathrm{hr}$ in the placebo group and $469 \pm 201 \mathrm{mg} / \mathrm{dL} \cdot 4 \mathrm{hr}$ in the LCE group. Figure 1A shows the increase of serum uric acid in all subjects and in subjects with a uric acid level of less than $7.0 \mathrm{mg} / \mathrm{dL}$. There were no significant differences up to $4 \mathrm{hr}$ after the test meal. Urinary excretion of uric acid was similar in both groups (Figure 1B).

\section{Four-week repeated-administration study}

Two subjects dropped out from each of the placebo and LCE groups during the study period for personal reasons. Table 1 shows the changes of serum uric acid after ingestion of placebo capsules or LSE capsules. Ingestion of LSE caused uric acid to decrease significantly $(-0.18 \mathrm{mg} /$ $\mathrm{dL})$. Statistical analysis was also performed in subjects with a uric acid level of more than 5.5 and less than $7.0 \mathrm{mg} / \mathrm{dL}$. As a result, significant reduction of the uric acid level was observed in LCE group compared to the placebo group. The changes of blood pressure, pulse rate, and urine $\mathrm{pH}$ (safety evaluation parameters) are indicated in Table 2. All values were in the normal ranges and significant differences were not detected between before and after ingestion or between the two groups. Moreover, inflammatory parameters (CRP, erythrocyte sedimentation rate, and white blood cell count) did not change during ingestion of placebo capsules or LCE capsules (Table 3).

\section{Discussion}

We investigated the effect of LCE on the serum uric acid level in healthy Japanese subjects. It was found that a single oral dose of LCE did not suppress the increase of uric acid after a high purine base test meal. Ueda et al. reported that chrysanthemum flower oil (CFO) containing luteolin suppressed elevation of serum uric acid in Japanese subjects after loading with a high purine base diet [22]. In their study, significant suppression of uric acid by CFO was observed in the subjects with a baseline serum uric acid level of $7.1 \mathrm{mg} / \mathrm{dL}$ or more, while there was no significant difference between the placebo and CFO groups in subjects with a baseline serum uric acid of $6.5 \mathrm{mg} / \mathrm{dL}$ or more. In Japan, the criterion for diagnosis of hyperuricemia is a serum uric acid level of more than $7.0 \mathrm{mg} / \mathrm{dL}$. In this study, we examined the effect of LCE on Japanese men with serum uric acid levels over the range from normal to hyperuricemia ( 5.5 to $8.0 \mathrm{mg} / \mathrm{dL}$ ). However, LCE did
Table 1. Serum uric acid level before and after ingestion of placebo or LCE for 4 weeks.

\begin{tabular}{|c|c|c|c|c|}
\hline & & Before & After & Net change $(\Delta)$ \\
\hline \multicolumn{5}{|l|}{ First period } \\
\hline Group A & Placebo & $5.84 \pm 0.94$ & $5.88 \pm 0.94$ & $0.05 \pm 0.55$ \\
\hline Group B & LCE & $6.12 \pm 1.10$ & $5.98 \pm 1.07$ & $-0.14 \pm 0.37$ \\
\hline \multicolumn{5}{|c|}{ Second period } \\
\hline Group A & LCE & $6.08 \pm 1.27$ & $5.82 \pm 1.02$ & $-0.26 \pm 0.50$ \\
\hline Group B & Placebo & $5.88 \pm 0.93$ & $5.65 \pm 0.88 \dagger$ & $-0.23 \pm 0.38$ \\
\hline \multicolumn{5}{|c|}{ Total (All subjects) } \\
\hline Placebo & & $5.96 \pm 1.10$ & $5.85 \pm 0.96$ & $-0.11 \pm 0.54$ \\
\hline LCE & & $6.00 \pm 1.00$ & $5.82 \pm 0.97 \dagger$ & $-0.18 \pm 0.37$ \\
\hline \multicolumn{5}{|c|}{ Total (uric acid of 5.5 to $7.0 \mathrm{mg} / \mathrm{dL}$ ) } \\
\hline Placebo & & $5.91 \pm 0.59$ & $6.09 \pm 0.52$ & $0.18 \pm 0.48$ \\
\hline LCE & & $6.18 \pm 0.65$ & $5.98 \pm 0.55$ & $-0.20 \pm 0.38^{*}$ \\
\hline
\end{tabular}

Values represent the mean and SD $(n=13$ or 26). Significant differences between placebo and LCE*: $p<0.05$. Significant difference between before and after ingestion $\dagger: \mathrm{P}<0.05$.

Table 2. Blood pressure, pulse rate, and urine $\mathrm{pH}$ before and after ingestion of LCE or placebo.

\begin{tabular}{|c|c|c|c|c|c|c|c|c|c|c|}
\hline \multirow{3}{*}{$\begin{array}{l}\text { Systolic blood } \\
\text { pressure } \\
(\mathrm{mmHg})\end{array}$} & & \multicolumn{3}{|c|}{ Before } & \multicolumn{3}{|c|}{ After 4 weeks } & \multicolumn{3}{|c|}{ Net change $(\Delta)$} \\
\hline & Placebo & 136.2 & \pm & 13.1 & 132.8 & \pm & 15.8 & -3.4 & \pm & 14.5 \\
\hline & LCE & 134.5 & \pm & 13.7 & 130.5 & \pm & 15.7 & -3.9 & \pm & 10.5 \\
\hline \multirow{2}{*}{$\begin{array}{l}\text { Diastolic } \\
\text { blood pressure } \\
(\mathrm{mmHg})\end{array}$} & Placebo & 81.5 & \pm & 8.6 & 79.6 & \pm & 8.9 & -1.9 & \pm & 8.1 \\
\hline & LCE & 80.4 & \pm & 11.7 & 79.2 & \pm & 10.0 & -1.3 & \pm & 9.9 \\
\hline \multirow{2}{*}{$\begin{array}{l}\text { Pulse rate } \\
\text { (/ min })\end{array}$} & Placebo & 78.1 & \pm & 17.9 & 77.8 & \pm & 13.3 & -0.3 & \pm & 10.5 \\
\hline & CFE & 73.8 & \pm & 14.2 & 79.2 & \pm & 10.0 & 5.4 & \pm & 14.5 \\
\hline \multirow{2}{*}{ Urine $\mathrm{pH}$} & Placebo & 6.63 & \pm & 0.48 & 6.67 & \pm & 0.49 & 0.04 & \pm & 0.47 \\
\hline & CFE & 6.52 & \pm & 0.54 & 6.69 & \pm & 0.49 & 0.17 & \pm & 0.45 \\
\hline
\end{tabular}

Values represent the mean and SD $(n=26)$. No significant differences were detected between before and after ingestion or between the two groups.

not suppress the increase of uric acid in all subjects or in the subjects with uric acid levels of less or more than $7.0 \mathrm{mg} / \mathrm{dL}$ (data not shown). Hence, CFO seems to reduce the postprandial uric acid level in patients with hyperuricemia, but LCE has no effect on postprandial elevation of uric acid. Differences in the constituents of CFO and LCE may have contributed to the differing results. The flavonoid content of CFO was not mentioned in the report, but the luteolin content of LCE is 10\% and the content of other polyphenolic compounds is relatively lower. Thus, luteolin (a principal component of LCE) does not seem to affect to postprandial elevation of the serum uric acid level.

After ingestion of LCE for 1 month in the second study, the serum uric acid level decreased in the subjects with a baseline uric acid level of 5.5 to $7.0 \mathrm{mg} / \mathrm{dL}$. Hence, LCE seems to suppress mildly elevated uric acid levels. Among structurally similar flavonoid derivatives, consumption of quercetin $(500 \mathrm{mg} /$ day) for 4 weeks has been reported to decrease the baseline serum uric acid level without affecting uric acid excretion [23]. In addition, 6-month consumption of soy bean-derived daizein (40 mg/day) decreased serum uric acid in hypercholesterolemic subjects [24] Among processed foods containing polyphenols, green tea [25] and black tea [26] extracts have been reported to suppress the serum uric acid level. Therefore, there is evidence that polyphenolic compounds like flavonoids and catechin derivatives may reduce the baseline serum uric acid level. Thus, luteolin in LCE might also have suppressed baseline serum uric acid by a similar mechanism to that reported for polyphenols.

\section{Conclusion}

Ingestion of LCE for 4 weeks suppressed the fasting serum uric acid level in Japanese men with mild hyperuricemia. 
Table 3. Laboratory parameters before and after ingestion of LCE or placebo.

\begin{tabular}{|c|c|c|c|c|c|c|c|c|c|c|c|c|c|c|c|c|c|c|c|}
\hline \multirow{3}{*}{$\begin{array}{c}\text { Parameter } \\
\text { C-reactive protein }\end{array}$} & \multirow{3}{*}{$\begin{array}{c}\text { Unit } \\
\mathrm{mg} / \mathrm{dL}\end{array}$} & \multicolumn{9}{|c|}{ Placebo } & \multicolumn{9}{|c|}{ LCE } \\
\hline & & \multicolumn{3}{|c|}{ Before } & \multicolumn{3}{|c|}{ After } & \multicolumn{3}{|c|}{ Net change $(\Delta)$} & \multicolumn{3}{|c|}{ Before } & \multicolumn{3}{|c|}{ After } & \multicolumn{3}{|c|}{ Net change $(\Delta)$} \\
\hline & & 0.17 & \pm & 0.35 & 0.09 & \pm & 0.08 & -0.08 & \pm & 0.33 & 0.10 & \pm & 0.15 & 0.12 & \pm & 0.02 & 0.01 & \pm & 0.13 \\
\hline Erythrocyte sedimentation rate ( 1 hour) & $\mathrm{mm}$ & 4.7 & \pm & 4.2 & 4.9 & \pm & 4.6 & 0.2 & \pm & 3.8 & 4.4 & \pm & 4.1 & 4.6 & \pm & 0.8 & 0.2 & \pm & 1.4 \\
\hline Erythrocyte sedimentation rate ( 2 hours) & $\mathrm{mm}$ & 12.4 & \pm & 11.1 & 13.2 & \pm & 11.8 & 0.8 & \pm & 8.9 & 11.0 & \pm & 10.4 & 12.4 & \pm & 1.9 & 1.4 & \pm & 3.5 \\
\hline White blood cell count & $\times 10^{2} / \mu \mathrm{L}$ & 61.5 & \pm & 14.4 & 56.0 & \pm & 12.7 & -5.5 & \pm & 14.6 & 59.1 & \pm & 15.3 & 59.6 & \pm & 2.7 & 0.5 & \pm & 18.7 \\
\hline
\end{tabular}

Values represent the mean and SD $(\mathrm{n}=26)$. No significant differences were detected between before and after ingestion or between the two groups.

A

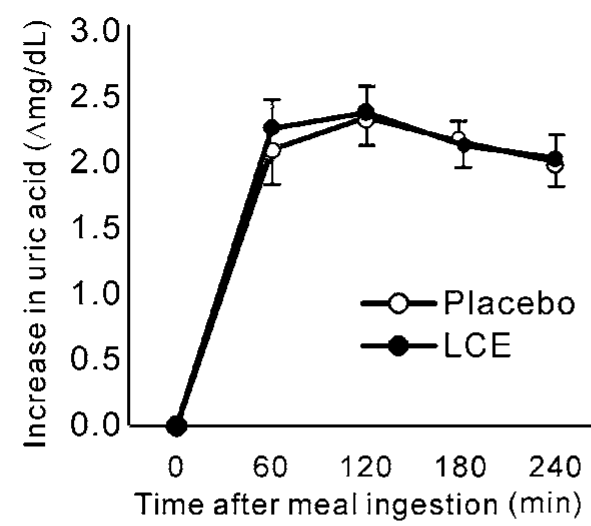

B

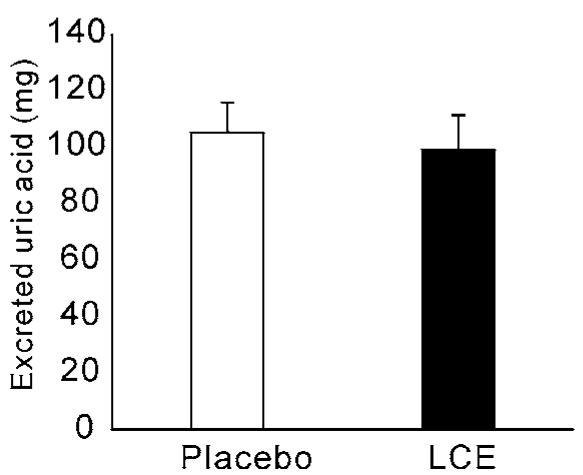

Subjects with uric acid less than $7.0 \mathrm{mg} / \mathrm{dL}$
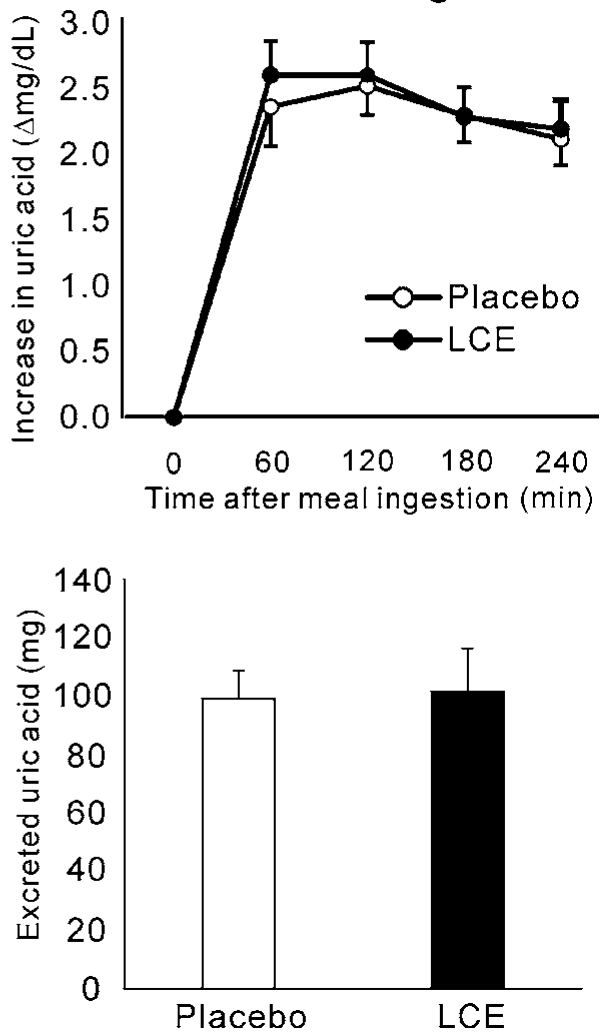

Values represent the mean and SD $(n=20)$. There were no significant differences between the LCE and placebo groups.

Figure 1. Change of serum uric acid after a single oral dose of LCE in subjects given a high purine base test meal.

\section{Funding}

The study was funded by Oryza Oil \& Fat Chemical Co. Ltd.

\section{Conflict of interest}

All authors related to this study are employees of Oryza Oil \& Fat Chemical Co., Ltd. (Aichi, Japan). The authors declare no conflict of interest associated with this manuscript.

\section{References}

1. Dalbeth N, Merriman TR, Stamp LK (2016) Gout. Lancet 388: 2039-2052. [Crossref]

2. Janssens HJ, Janssen M, van de Lisdonk EH, van Riel PL, van Weel C (2008) Use of oral prednisolone or naproxen for the treatment of gout arthritis: a double-blind, randomised equivalence trial. Lancet 371: 1854-1860. [Crossref]

3. Rainer TH, Cheng CH, Janssens HJ, Man CY, et al. (2016) Oral prednisolone in the treatment of acute gout: a pragmatic, multicenter, double-blind, randomized trial. Ann Intern Med 164: 464-471. [Crossref]

4. Wang Y, Wang L, Li E, Li Y, Wang Z, et al. (2014) Chuanhu anti-gout mixture versus colchicine for acute gouty arthritis: a randomized, double-blind, double-dummy, noninferiority trial. Int J Med Sci 11: 880-885. [Crossref]
5. Takir M, Kostek O, Ozkok A, Elcioglu OC, Bakan A, et al. (2015) Lowering uric acid with allopurinol improves insulin resistance and systemic inflammation in asymptomatic hyperuricemia. J Investig Med 63: 924-929.

6. Kaneko K, Aoyagi Y, Fukuuchi T, Inazawa K, Yamaoka N (2014) Total purine and purine base content of common foodstuffs for facilitating nutritional therapy for gout and hyperuricemia. Biol Pharm Bull 37: 709-721

7. Inokuchi T, Ka T, Yamamoto A, Takahashi S, Tsutsumi Z, et al. (2008) Effects of allopurinol on beer-induced increases in plasma concentrations of purine bases and uridine. Nucleosides Nucleotides Nucleic Acids 27: 601-603. [Crossref]

8. Hakoda M (2012) Recent trends in hyperuricemia and gout in Japan. Japan Med Assoc J 55: 319-323. [Crossref]

9. Kong LD, Cai Y, Huang WW, Cheng CH, Tan RX (2000) Inhibition of xanthine oxidase by some Chinese medicinal plants used to treat gout. J Ethnopharmacol 73: 199-207. [Crossref]

10. Akihisa T, Yasukawa K, Oinuma H, Kasahara Y, Yamanouchi S, et al. (1996) Triterpene alcohols from the flowers of compositae and their anti-inflammatory effects. Phytochemistry 43: 1255-1260. [Crossref]

11. Ukiya M, Akihisa T, Yasukawa K, Kasahara Y, Kimura Y, et al. (2001) Constituents of compositae plants. 2. Triterpene diols, triols, and their 3-O-fatty acid esters from edible chrysanthemum flower extract and their anti-inflammatory effects. $J$ Agric Food Chem 49: 3187-3197. [Crossref] 
12. Luyen BT, Tai BH, Thao NP, Cha JY, Lee HY, et al. (2015) Anti-inflammatory components of Chrysanthemum indicum flowers. Bioorg Med Chem Lett 25: 266-269. [Crossref]

13. Chen XY, Li J, Cheng WM, Jiang H, Xie XF, et al. (2008) Effect of total flavonoids of Chrysanthemum indicum on the apoptosis of synoviocytes in joint of adjuvant arthritis rats. Am J Chin Med 36: 695-704. [Crossref]

14. Honda S, Kawamoto S, Tanaka H, Kishida H, Kitagawa M, et al. (2014) Administered chrysanthemum flower oil attenuates hyperuricemia: mechanism of action as revealed by DNA microarray analysis. Biosci Biotechnol Biochem 78: 655-661. [Crossref]

15. Chen L, Kotani A, Kusu F, Wang Z, Zhu J, et al. (2015) Quantitative comparison of caffeoylquinic acids and flavonoids in Chrysanthemum morifolium flowers and their sulfur-fumigated products by three-channel liquid chromatography with electrochemical detection. Chem Pharm Bull (Tokyo) 63: 25-32. [Crossref]

16. Yan J, Zhang G, Hu Y, Ma Y (2013) Effect of luteolin on xanthine oxidase: inhibition kinetics and interaction mechanism merging with docking simulation. Food Chem 141: 3766-3773. [Crossref]

17. Li Y, Frenz CM, Li Z, Chen M, Wang Y, et al. (2013) Virtual and in vitro bioassay screening of phytochemical inhibitors from flavonoids and isoflavones against xanthine oxidase and cyclooxygenase-2 for gout treatment. Chem Biol Drug Des 81: 537-544. [Crossref]

18. Kong LD, Wolfender JL, Cheng CH, Hostettmann K, Tan RX (1999) Xanthine oxidase inhibitors from Brandisia hancei. Planta Med 65: 744-746. [Crossref]
19. de Souza MR, de Paula CA, Pereira de Resende ML, Grabe-Guimarães A, de Souza Filho JD, et al. (2012) Pharmacological basis for use of Lychnophora trichocarpha in gouty arthritis: anti-hyperuricemic and anti-inflammatory effects of its extract, fraction and constituents. J Ethnopharmacol 142: 845-850. [Crossref]

20. Mo SF, Zhou F, Lv YZ, Hu QH, Zhang DM, et al. (2007) Hypouricemic action of selected flavonoids in mice: structure-activity relationships. Biol Pharm Bull 30: 15511556. [Crossref]

21. Ding Y, Shi X, Shuai X, Xu Y, Liu Y, et al. (2014) Luteolin prevents uric acid-induced pancreatic $\beta$-cell dysfunction. J Biomed Res 28: 292-298. [Crossref]

22. Ueda T, Honda S, Morikawa H, Kitamura S, Iwama Y, et al. (2015) Chrysanthemum flower oil inhibits diet-induced serum uric acid elevation in adult male subjects. A randomized, double-blind, placebo-controlled cross-over comparison study. Nutrafoods 14: $151-158$.

23. Shi Y, Williamson G (2016) Quercetin lowers plasma uric acid in pre-hyperuricaemic males: a randomized, double-blinded, placebo-controlled, cross-over trial. Br J Nutr 115(5): 800-6. [Crossref]

24. Kimira M, Arai Y, Shimoi K, Watanabe S (1998) Japanese intake of flavonoids and isoflavonoids from foods. J Epidemiol 8: 168-175. [Crossref]

25. Jatuworapruk K, Srichairatanakool S, Ounjaijean S, Kasitanon N, Wangkaew S, et al. (2014) Effects of green tea extract on serum uric acid and urate clearance in healthy individuals. J Clin Rheumatol 20: 310-313. [Crossref]

26. Bahorun T, Luximon-Ramma A, Gunness TK, Sookar D, Bhoyroo S, et al. (2010) Black tea reduces uric acid and C-reactive protein levels in humans susceptible to cardiovascular diseases. Toxicology 278: 68-74. [Crossref]

Copyright: $(02017$ Hirano M. This is an open-access article distributed under the terms of the Creative Commons Attribution License, which permits unrestricted use, distribution, and reproduction in any medium, provided the original author and source are credited. 